\title{
Memory effects in classical and quantum mean-field disordered models
}

\author{
L. F. Cugliandolo ${ }^{1,2}$, G. Lozano ${ }^{3}$ and H. Lozza ${ }^{3}$ \\ ${ }^{1}$ Laboratoire de Physique Théorique de l'École Normale Supérieure, \\ 24 rue Lhomond, 75231 Paris Cedex 05, France \\ ${ }^{2}$ Laboratoire de Physique Théorique et Hautes Énergies, Jussieu, \\ 1er étage, Tour 16, 4 Place Jussieu, 75252 Paris Cedex 05, France \\ ${ }^{3}$ Departamento de Física, FCEyN, Universidad de Buenos Aires, \\ Pabellón I, Ciudad Universitaria, 1428 Buenos Aires, Argentina
}

November 19, 2018

\begin{abstract}
We apply the Kovacs experimental protocol to classical and quantum $p$ spin models. We show that these models have memory effects as those observed experimentally in super-cooled polymer melts. We discuss our results in connection to other classical models that capture memory effects. We propose that a similar protocol applied to quantum glassy systems might be useful to understand their dynamics.
\end{abstract}




\section{Introduction}

The Kovacs memory effect was first reported by this author in the 60s [1]. It demonstrates that super-cooled liquids when taken out of equilibrium have a very intricate dynamics that cannot be predicted on the basis of the knowledge of the instantaneous value of the state variables $(P, V, T)$ right after the perturbation. More precisely, Kovacs showed that the specific volume of a polymer melt in its supercooled liquid phase, has a rather non-trivial evolution that depends on the thermal history of the sample [1, 2]. Non-trivial effects of temperature variations were also observed in the evolution of (two-time) susceptibilities of dipolar glasses [3], spinglasses [4] and many other glassy systems [5, 6]. History-dependent phenomena in granular compaction have been recently reported [7]. In this case, the control parameter is the tapping strength and the observable is the density.

The experimental setup involved in the Kovacs effect is the following. In a first step, one quenches an equilibrated liquid with a very fast cooling rate from a high temperature $T_{0}$ to a low temperature $T_{2}$, at time $t=0$. One then follows the subsequent evolution of the quantity of interest that in Kovacs' experiments is the specific volume. In order to match what we shall study in this paper, we describe the experiment using another one-time quantity, the energy density, as the example. The energy density relaxes in time and it slowly approaches an asymptotic value that may fall out of the experimental time-window. The time-dependent curve $\mathcal{E}^{\left(T_{2}\right)}(t)$ constitutes a reference and we use a superscript $\left(T_{2}\right)$ to indicate that it has been obtained using the first prescription, at fixed external temperature $T_{2}$. The extrapolated asymptotic value defines $\mathcal{E}_{a s}\left(T_{2}\right)$. In a second step, one first quenches the sample from the same high temperature $T_{0}$ to a lower temperature $T_{1}\left(<T_{2}\right)$ at time $t=0$, waits until a carefully chosen time $t_{1}$ and then heats the sample

to the final temperature $T_{2}$. The value of $t_{1}$ is chosen such that $\mathcal{E}^{\left(T_{1} \rightarrow T_{2}\right)}\left(t_{1}^{+}\right)=$ $\mathcal{E}_{a s}\left(T_{2}\right)$, where the plus sign indicates that the one-time quantity should match the asymptotic value obtained with the first procedure right after heating the sample from $T_{1}$ to $T_{2}$. [In the experimental protocol the need to use $\mathcal{E}^{\left(T_{1} \rightarrow T_{2}\right)}\left(t_{1}^{+}\right)=\mathcal{E}_{a s}\left(T_{2}\right)$ is due to the fact that when changing the temperature there is a trivial response due to the thermal expansion of the local degrees of freedom.] Since the initial value of the energy density at the final temperature $T_{2}$ is already the asymptotic one, one could have expected that the energy remained constantly fixed to this value for all subsequent times independently of the value of $T_{1}$ (apart from very fast rearrangements decaying exponentially). However, Kovacs demonstrated that after 
$t_{1}$ the energy-density, $\mathcal{E}^{\left(T_{1} \rightarrow T_{2}\right)}(t)$, has a slow non-monotic dependence on time, first increasing and then decreasing back to its initial and asymptotic value $\mathcal{E}_{a s}\left(T_{2}\right)$ :

$$
\mathcal{E}^{\left(T_{1} \rightarrow T_{2}\right)}(t)=\mathcal{E}_{a s}\left(T_{2}\right)+\Delta \mathcal{E}(t)
$$

with the "Kovacs hump" $\Delta \mathcal{E}$ satisfying

$$
\Delta \mathcal{E}(t)>0, \quad \Delta \mathcal{E}\left(t_{1}^{+}\right)=\lim _{t \rightarrow \infty} \Delta \mathcal{E}(t)=0
$$

The form of the hump depends on the values $T_{2}$ and $T_{1}$ used. Qualitatively, its height is an increasing function of $T_{2}-T_{1}$ and the time at which the maximum is reached decreases when $T_{2}-T_{1}$ increases.

It is interesting to note that Kovacs' experiments have been performed in the super-cooled liquid phase. While it would be no surprise to find nonequilibrium effects in the glassy phase, the reason why one also finds a nonequilibrium behaviour in the super-cooled liquid is that the jump in the external temperature drives the system out of equilibrium and the relaxation occurs in a very long time-scale. This experiment proves that the knowledge of the state variables, in Kovacs' experiments $P, V$ and $T$ at $t_{1}^{+}$, is not sufficient to determine the subsequent evolution of the same quantities in the glassy and super-cooled liquid phases (if the latter has been recently strongly perturbed).

Recently, several authors presented analytical and numerical studies of this effect using a variety of models with glassy dynamics. So far, apart from phenomenological approaches [2], the Kovacs effect has been analysed numerically with molecular dynamic simulations of a molecular model of a fragile glass former [8] and Montecarlo simulations of the $3 d$ Edwards-Anderson spin-glass 9], and analytically within the ferromagnetic Ising chain [10], the critical $2 d$ XY model [1], the trap model [12], domain growth 12, 1d kinetically constrained spin models of fragile and strong type [13], and the parking lot model of granular matter [14]. It has been suggested that the quantitative analysis of the Kovacs effect may help distinguishing between different glassy models and, perhaps, may also help identifying spatial properties of glassy systems. In this paper we show that the main qualitative features of the classical Kovacs effect are captured by the fully connected spherical $p$-spin disordered system [15], a model with no spatial structure but with a slow dynamics leading to very slow relaxations close to the transition to and in the glassy phase [16, 17. This 'negative' result, as far as what can be deduced about spatial rearrangements from the Kovacs effect, is reminiscent of the discussion [18] on the interpretation of 
hole burning experiments [19]. We also discuss the scaling laws that describe the behaviour of the hump and compare them to what found in other glassy models.

On the other hand, the analysis of quantum glassy systems is now starting to call the attention of experimentalists and theoreticians. The slow, history-dependent relaxation of a dipolar quantum system in its glassy phase has been reported [20]. The sample that entered the glassy phase following a quantum route (changing the strength of quantum fluctuations at fixed low temperature) is always in advance with respect to the one that arrived at the same point in parameter space following a classical path (keeping the strength of quantum fluctuations fixed and cooling the system). This has been demonstrated by the fact that the time-dependent dielectric constant of the quantum-cooled sample is closer to its asymptotic value at any finite, experimental time. Memory effects were also observed in glasses at ultralow temperatures [21] and the electron glass [22].

A variant of the Kovacs procedure where the control parameter is the strength of quantum fluctuations can be easily envisaged. The question then arises as to whether a hump appears and which is its structure, scaling form, etc. We address this question using a quantum extension of the $p$-spin model introduced and studied in [23, 24] (see also [25]).

In short, in this paper we show that simple disordered mean-field models capture the phenomenology of the Kovacs effect. With this aim, we analyse the nonequilibrium relaxation of the spherical $p$-spin disordered model in its classical [16, 17] and quantum [23] versions, see Sect. 2 for their definitions. First, we reproduce the classical setup using temperature as the control parameter and we discuss the results in comparison with previous explanations of the same effect (Sect. 31). Second, we switch on quantum fluctuations and use their strength as the control parameter (Sect. 4). In both cases we follow the evolution in time of the potential energydensity of the system. We present our conclusions in Sect. 5 ,

\section{The spherical p-spin model}

The spherical $p$ spin model is defined by the Hamiltonian [15]

$$
H_{J}[\vec{S}]=-\sum_{\left\langle i_{1} i_{2} \ldots i_{p}\right\rangle} J_{i_{1} i_{2} \ldots i_{p}} s_{i_{1}} s_{i_{2}} \ldots s_{i_{p}}
$$

The spins $s_{i}$ are continuous variables $s_{i} \in(-\infty, \infty)$ forced to satisfy the global spherical constraint $\sum_{i=1}^{N} s_{i}=N$ with $N$ their total number in the sample. The 
exchange interaction $J_{i_{1} i_{2} \ldots i_{p}}$ are quenched random variables drawn from a Gaussian distribution with average $\left[J_{i_{1} \ldots i_{p}}\right]=0$ and variance $\left[J_{i_{1} \ldots i_{p}}^{2}\right]=\tilde{J}^{2} p ! /\left(2 N^{p-1}\right)$. We henceforth use square brackets to indicate the average over disorder. The interactions occur between all groups of $p$ spins in the sample. The model is then fully-connected and mean-field in character. The scaling of the variance $\left[J_{i_{1} \ldots i_{p}}^{2}\right]$ with $N$ has been chosen so as to ensure a good thermodynamic limit. The parameter $p$ takes integer values, $p \geq 2$. Mixed models with a Hamiltonian with two terms of the form (3) with $p=2$ and $p=4$ [26] are also of interest [17].

The classical dynamics is determined by the Langevin equation

$$
\dot{s}_{i}(t)=-\frac{\partial E(t)}{\partial s_{i}(t)}-\mu(t) s_{i}(t)+\xi_{i}(t)
$$

where $E(t)$ is the total energy, $\mu(t)$ is the Lagrange multiplier enforcing the spherical constraint, and $\xi_{i}(t)$ is a Gaussian white-noise with zero mean and correlations

$$
\left\langle\xi_{i}(t) \xi_{j}\left(t^{\prime}\right)\right\rangle=2 k_{B} T \delta_{i j} \delta\left(t-t^{\prime}\right) .
$$

We assume that the system is prepared at $t=0$ with an infinitely fast quench from $T_{0} \rightarrow \infty$ to the initial temperature $T_{1}$. The dynamic equations for the macroscopic order parameters are derived using standard functional techniques [17]. We discuss them below, as the classical limit of the quantum extension of the same model. The models with $p=2, p \geq 3$ have different dynamics; the former yields a mean-field description of simple domain growth, while the latter case is related to the schematic mode-coupling theory (MCT) of super-cooled liquids and glasses. These models and their generalizations are reviewed in detail in [17.

Quantum fluctuations can be introduced 23] by upgrading the spins $s_{i}$ to coordinatelike operators, adding a 'kinetic term'

$$
\frac{1}{2 M} \sum_{i=1}^{N} \hat{\Pi}_{i}^{2}
$$

to the Hamiltonian (3), and imposing canonical commutation relations

$$
\left[\hat{\Pi}_{i}, \hat{s}_{j}\right]=i \hbar \delta_{i j}, \quad\left[\hat{s}_{i}, \hat{s}_{j}\right]=0, \quad\left[\hat{\Pi}_{i}, \hat{\Pi}_{j}\right]=0 .
$$

At time $t=0$ we set the model in contact with an Ohmic bath of quantum harmonic oscillators

$$
\begin{aligned}
H_{b} & =\sum_{l=1}^{\tilde{N}} \frac{1}{2 m_{l}} \hat{p}_{l}^{2}+\sum_{l=1}^{\tilde{N}} \frac{1}{2} m_{l} \omega_{l}^{2} \hat{x}_{l}^{2}, \\
H_{s b} & =-\sum_{i=1}^{N} \hat{s}_{i}^{z} \sum_{l=1}^{N} c_{i l} \hat{x}_{l},
\end{aligned}
$$


where, for simplicity, we considered a bilinear coupling, $H_{s b}$. (Note that for the spherical problem it is not necessary to introduce a counterterm). We assume that this environment has a well-defined temperature and that it is not modified by the interaction with the system. The initial density matrix is factorised and we choose random initial conditions for the system. After integrating out the bath degrees of freedom, and using the fully-connected character of (3), one arrives at a dynamic generating functional from which one derives exact dynamic equations for the macroscopic two-time dependent order parameters

$$
C\left(t, t^{\prime}\right)=\frac{1}{N} \sum_{i=1}^{N}\left[\left\langle\left\{\hat{s}_{i}(t), \hat{s}_{i}\left(t^{\prime}\right)\right\}\right\rangle\right], \quad R\left(t, t^{\prime}\right)=\left.\frac{1}{N} \sum_{i=1}^{N} \frac{\left[\left\langle\hat{s}_{i}(t)\right\rangle\right]}{h_{i}\left(t^{\prime}\right)}\right|_{h=0} .
$$

$h$ is an infinitesimal field that couples linearly to the spins, modifying the Hamiltonian as $H \rightarrow H-\sum_{i=1} h_{i} \hat{s}_{i}$. $C$ is the symmetrized correlation function and $R$ is the linear response of the system.

The dynamic equations take the Schwinger-Dyson form

$$
\begin{aligned}
& {\left[M \partial_{t}^{2}+\mu(t)\right] R\left(t, t^{\prime}\right)=\delta\left(t-t^{\prime}\right)+\int_{t^{\prime}}^{t} d t^{\prime \prime} \Sigma\left(t, t^{\prime \prime}\right) R\left(t^{\prime \prime}, t^{\prime}\right),} \\
& {\left[M \partial_{t}^{2}+\mu(t)\right] C\left(t, t^{\prime}\right)=\int_{0}^{t} d t^{\prime \prime} \Sigma\left(t, t^{\prime \prime}\right) C\left(t^{\prime \prime}, t^{\prime}\right)+\int_{0}^{t^{\prime}} d t^{\prime \prime} D\left(t, t^{\prime \prime}\right) R\left(t^{\prime}, t^{\prime \prime}\right),}
\end{aligned}
$$

with the self-energy $\Sigma$ and the vertex $D$ given by

$$
\begin{aligned}
& \Sigma\left(t, t^{\prime \prime}\right)=-4 \eta\left(t-t^{\prime}\right)+\sigma\left(t, t^{\prime}\right) \\
& D\left(t, t^{\prime \prime}\right)=2 \hbar \nu\left(t-t^{\prime}\right)+d\left(t, t^{\prime}\right) .
\end{aligned}
$$

The first contributions originate in the interaction with the Ohmic bath of spectral density [23, 24]

$$
I(\omega)=\frac{4 \gamma}{\pi} \omega e^{-\omega / \Lambda} \theta(\omega)
$$

where $\Lambda$ is a cut-off included to avoid divergences. The kernels $\eta$ and $\nu$ are functions of the time-difference $\tau \equiv t-t^{\prime}$ and they are given by

$$
\begin{aligned}
\eta(\tau) & =-\frac{4 \gamma \Lambda^{2}}{\pi} \frac{2 \Lambda \tau}{\left(1+(\Lambda \tau)^{2}\right)^{2}} \\
\nu(\tau) & =\frac{4 \gamma}{\pi} \int_{0}^{\infty} d \omega \omega e^{-\omega / \Lambda} \operatorname{coth}\left(\frac{\beta \hbar \omega}{2}\right) \cos (\omega \tau)
\end{aligned}
$$

The second contributions, $\sigma$ and $d$, are due to the interactions in the system and read

$$
\sigma\left(t, t^{\prime}\right) \equiv-\frac{p \tilde{J}^{2}}{\hbar} \operatorname{Im}\left[C\left(t, t^{\prime}\right)-\frac{i \hbar}{2} R\left(t, t^{\prime}\right)\right]^{p-1}
$$




$$
d\left(t, t^{\prime}\right) \equiv \frac{p \tilde{J}^{2}}{2} \operatorname{Re}\left[C\left(t, t^{\prime}\right)-\frac{i \hbar}{2}\left(R\left(t, t^{\prime}\right)+R\left(t^{\prime}, t\right)\right)\right]^{p-1} .
$$

An integral equation that fixes the Lagrange multiplier $\mu(t)$ supplements these equations and it is derived from the requirement $C(t, t)=1$ for all times 23 .

The classical limit is easily obtained by neglecting the kinetic term and by taking the limit $\hbar \rightarrow 0$. Indeed, in this case, the effect of the coupling to the bath reduces to the usual contributions originating in the friction and noise terms of the Langevin equation and involving a first-order time-derivative of the correlation and response when $\Lambda$ is further taken to infinity.

In the quantum case, three contributions to the total energy density can be identified: the kinetic part, the potential part and the interaction with the bath. In the following we focus on the averaged potential energy density

$$
\mathcal{E}(t) \equiv N^{-1}\left[\left\langle H_{J}[\vec{S}]\right\rangle\right]=-\frac{1}{N}\left[\left\langle\sum_{\left\langle i_{1} i_{2} \ldots i_{p}\right\rangle} J_{i_{1} i_{2} \ldots i_{p}} \hat{s}_{i_{1}}(t) \hat{s}_{i_{2}}(t) \ldots \hat{s}_{i_{p}}(t)\right\rangle\right]
$$

With a simple calculation one finds

$$
\mathcal{E}(t)=\int_{0}^{\infty} d t^{\prime}\left[\sigma\left(t, t^{\prime}\right) C\left(t, t^{\prime}\right)+d\left(t, t^{\prime}\right) R\left(t, t^{\prime}\right)\right]
$$

that in the classical case becomes

$$
\mathcal{E}(t)=-\frac{\tilde{J}^{2} p}{2} \int_{0}^{t} d t^{\prime} C^{p-1}\left(t, t^{\prime}\right) R\left(t, t^{\prime}\right)
$$

\section{Classical case}

In this Section we analyze the classical problem with $p=3$. After recalling the value of the asymptotic energy-density for the isothermal relaxation, we solve numerically the dynamic equations for $C$ and $R$ following Kovacs' protocol. We analyse the Kovacs hump and we obtain its scaling with time and temperature in the three regimes of short, intermediate and long times.

\subsection{Analytic results}

In the high-temperature phase the Fluctuation Dissipation Theorem (FDT) implies

$$
\mathcal{E}_{a s}(T) \equiv-\frac{p}{2} \lim _{t \rightarrow \infty} \int_{0}^{t} d t^{\prime} C^{p-1}\left(t, t^{\prime}\right) \frac{1}{T} \frac{\partial C\left(t, t^{\prime}\right)}{\partial t^{\prime}}=-\frac{1}{2 T}
$$


$k_{B}=\tilde{J}=1$ henceforth. We shall focus on the $p=3$ model and use $T_{2}=0.75$ as the classical reference case for which $\mathcal{E}_{a s}\left(T_{2}\right) \approx-0.67$ (see Fig. 11).

This model undergoes a dynamic transition from an equilibrium (paramagnetic) to a nonequilibrium (glassy) phase at

$$
T_{d}=\sqrt{\frac{p(p-2)^{p-2}}{2(p-1)^{p-1}}} .
$$

For $p=3, T_{d} \approx 0.61$ and the asymptotic energy-density at the dynamic critical temperature takes the value $\mathcal{E}_{a s}\left(T_{d}\right) \approx-0.82$.

In the low-temperature phase the solution of equations (11)-(19) involves a modification of the FDT [16, 17], $R_{s}\left(t, t^{\prime}\right)=T_{e f f}^{-1} \partial_{t^{\prime}} C_{s}\left(t, t^{\prime}\right) \theta\left(t-t^{\prime}\right)$, for the slow part of the relaxation. The effective temperature [27] is given by $T_{\text {eff }}=\left(q_{e a} T\right) /\left[(p-2)\left(1-q_{e a}\right)\right]$ and the energy-density reads

$$
\mathcal{E}_{a s}(T)=-\frac{1}{2 T}\left[(p-2)\left(1-q_{e a}\right) q_{e a}^{p-1}+\left(1-q_{e a}^{p}\right)\right]
$$

with the Edwards-Anderson order parameter, $q_{e a}$, determined by

$$
\frac{p(p-1)}{2} q_{e a}^{p-2}\left(1-q_{e a}\right)^{2}=T^{2} .
$$

At the dynamic transition $T_{e f f}=T, q_{d}=(p-2) /(p-1)$ and $T_{d}$ is given by Eq. (24). The numerical solution of Eqs. (25) and (26) yields the value of the asymptotic energy-density in the glassy phase.

\section{$3.2 \quad$ Numerical results}

The effect of temperature variations on the dynamics of the $p$ spin models in the glassy phase was studied in a couple of papers. The effect of small amplitude temperature cycles on the nonequilibrium relaxation of the $p=2$ model were discussed in [28] while their influence on the dynamics of the $p=3$ was analyzed in [29].

We show here that the $p$ spin model with $p \geq 2$ captures a similar phenomenology in the sense that a hump with a slow relaxation is obtained when the Kovacs' protocol is applied. The model with $p=2$ is related to ferromagnetic domain growth, as described by the $O(N)$ model in $d=3$, and our results are intimately related to the ones in 10, 12. In the following we present the data for $p=3$ (the schematic mode-coupling-theory [17, 26]) only.

Figure 1 shows the time-evolution of the energy-density in the $p=3$ classical model using different temperature jumps chosen according to Kovacs' rule. The 


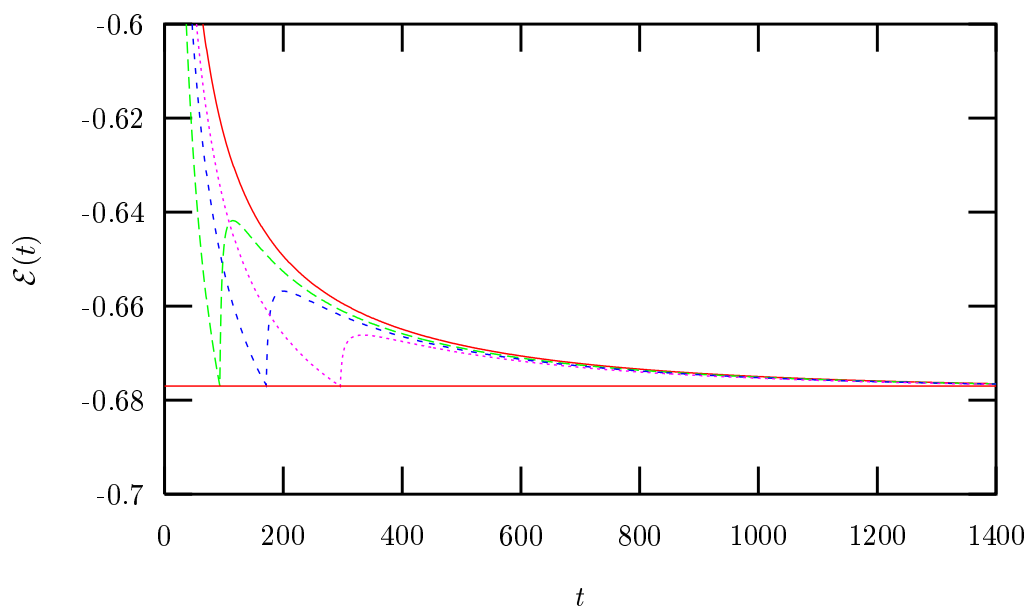

Figure 1: The evolution in time of the energy-density $\mathcal{E}(t)$ in the classical $p=3$ spin model close and above the paramagnetic - spin-glass transition. The solid line is $\mathcal{E}^{\left(T_{2}\right)}(t)$ and has been obtained using a rapid quench to $T_{2}=0.75$. The other curves show $\mathcal{E}^{\left(T_{1} \rightarrow T_{2}\right)}(t)$, i.e. the result of having performed a temperature jump from $T_{1}=0.65,0.7,0.725$ to $T_{2}$ at $t_{1}=92,170,295$ time-steps, respectively. The asymptotic value $\mathcal{E}_{a s}\left(T_{2}\right) \approx-0.677$ is shown with a horizontal line.

solid line has been obtained using a quench to $T_{2}=0.75$. Note that since $T_{2}>T_{d}$ the quench is done within the paramagnetic phase in which the equilibration time is finite. The asymptotic value approached with the algorithm is $\mathcal{E}_{a s}\left(T_{2}\right) \approx-0.677$. (The analytic prediction is $\mathcal{E}_{a s}\left(T_{2}\right) \approx-0.667$. Here and in what follows we use a time step $\delta=0.05$ to numerically solve the integro-differential equations.) The other curves include a temperature jump from $T_{1}$ to $T_{2}$ at $t_{1}$ when $\mathcal{E}^{\left(T_{1} \rightarrow T_{2}\right)}\left(t_{1}\right)=$ $\mathcal{E}_{a s}\left(T_{2}\right)$. The values of the parameters $T_{1}$ and $t_{1}$ are given in the caption. As observed by Kovacs experimentally [1] and for a variety of models [8] - [14], the evolution of the energy-density is non-monotonic. Since the time $t_{1}$ is chosen so as to have $\mathcal{E}^{T_{1} \rightarrow T_{2}}\left(t_{1}^{+}\right)=\mathcal{E}_{a s}\left(T_{2}\right) \approx-0.677$, after the temperature jump the energy density already has the expected asymptotic value. However, it evolves during approximately three decades before reaching the reference curve, first increasing towards a maximum and then decreasing towards $\mathcal{E}_{\text {as }}\left(T_{2}\right)$.

The qualitative features of this non-monotonic behaviour are better described by analysing the hump $\Delta \mathcal{E}$. Figure 2 shows its dependence with the temperature difference $T_{2}-T_{1}$. As in the experimental data, the height of the hump, $\Delta \mathcal{E}_{K} \equiv$ max $\Delta \mathcal{E}$, is an increasing function of $x \equiv T_{2}-T_{1}$, while the position of the maximum, $\left.t_{K} \equiv t\right|_{\max }$, is a decreasing function of the same parameter. In Fig. [3 we show that 


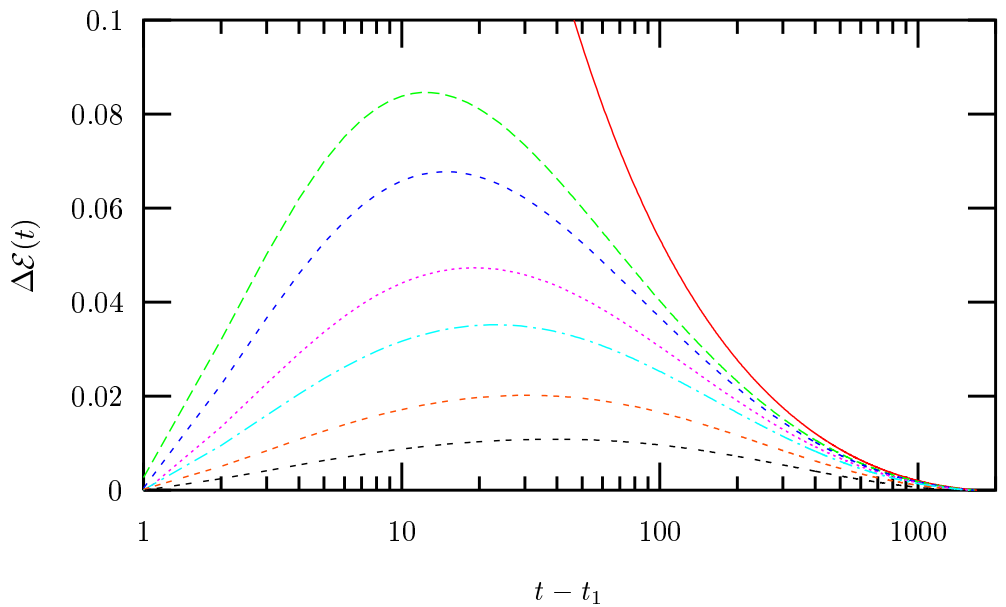

Figure 2: The hump, $\Delta \mathcal{E}(t)$, as a funtion of the time-difference, $t-t_{1}$, in the classical $p$ spin model with $p=3$ close and above its paramagnetic - spin-glass transition. The different curves correspond to the intermediate temperatures $T_{1}=0.4,0.5,0.6$, $0.65,0.7,0.725$ (from top to bottom) and the reference curve has been obtained for $T_{2}=0.75$.

these quantities are well described by functions of the type

$$
\begin{aligned}
\Delta \mathcal{E}_{K}(x) & =a x(1-b \ln x) \\
t_{K}(x) & =\frac{c}{x}(1-d \ln x)
\end{aligned}
$$

with $a, b, c, d$ fitting parameters.

We also show in Fig. 4 that $t_{K}$, the position of the maximum, can be fitted as $t_{K}=a t_{1}^{b}$, where $t_{1}$ is the time when the temperature jump has been applied, and $a$ and $b$ are constants.

Three time-regimes can be identified in the hump: short times well before the maximum is reached, intermediate times around the maximum and long-times when the hump approaches its asymptotic vanishing value. The time-temperature dependence in the three regimes can be summarized as follows.

\section{$\underline{\text { Short times }}$}

In the short time regimen, a linear function with logarithmic corrections [12]

$$
\mathcal{F}_{s}(x)=a x(1-b \ln x)
$$

fits the data with great precition. Indeed, in Fig. 5 we show together the data for $T_{1}=0.6,0.65,0.7$ and $t_{1}=63,92,170$ respectively and the fits by $\mathcal{F}_{s}(x)$. 


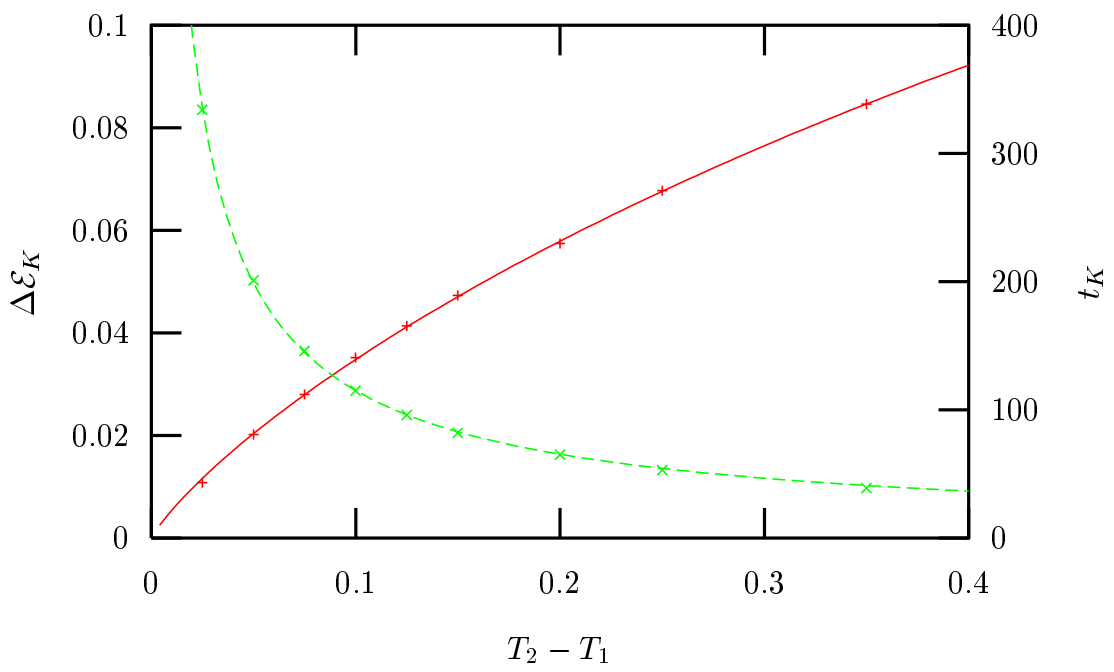

Figure 3: The maximum height of the hump, $\Delta \mathcal{E}_{K}(t)=\max \Delta \mathcal{E}$ (red curve, + ) and its position $\left.t_{K} \equiv t\right|_{\max }$ (green curve, $\times$ ) as a funtion of the difference in temperature $T_{2}-T_{1}$ in the classical $p$ spin model with $p=3$ at $T_{2}=0.75$. The lines are fits to power laws with logarithmic corrections, see Eqs. (27) and (28), with parameters $a=0.153$ and $b=0.556$ for the height of the maximum and $c=16.73$ and $d=-0.135$ for its position.

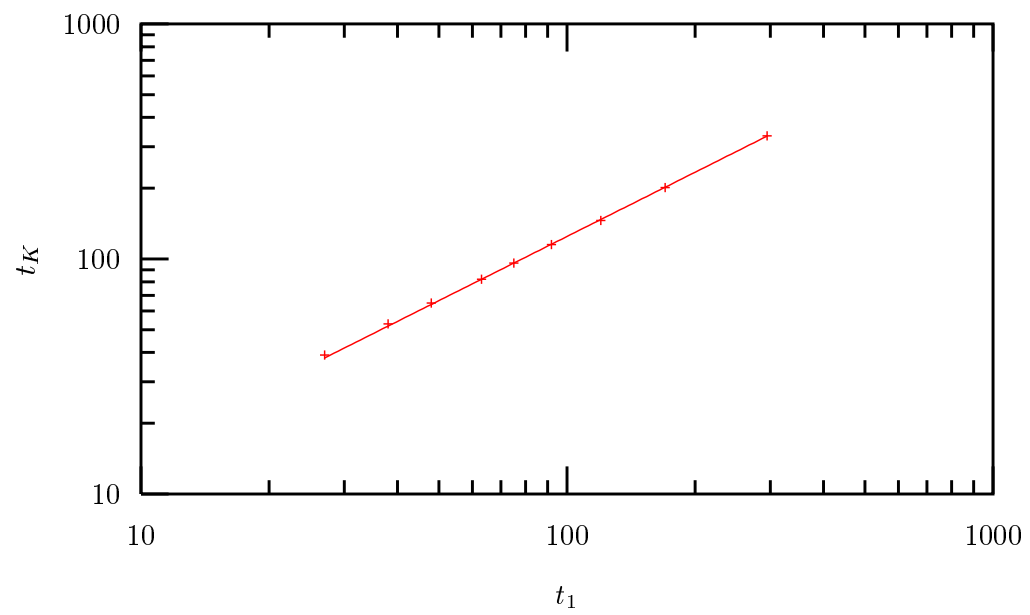

Figure 4: The position of the maximum in the hump, $\left.t_{K} \equiv t\right|_{\max }$, as a function of the time when the jump in temperature is imposed, $t_{1}$. The line is the power-law relation $t_{K}=a t_{1}^{b}$ with $a=1.901$ and $b=0.908$. 
The data can also be scaled using

$$
\Delta \mathcal{E}(t)=\left(T_{2}-T_{1}\right) \mathcal{F}_{s}\left(\left(t-t_{1}\right) \frac{T_{1}}{T_{2}}\right) .
$$

An accurate description of the rescaled data is achieved taking $a=0.0623$ and $b=0.251$ for $\mathcal{F}_{s}(x)$, see Fig. [6]

\section{Intermediate times}

The time-temperature dependence of the intermediate part of the relaxation is well described with the scaling form

$$
\Delta \mathcal{E}(t)=\Delta \mathcal{E}_{K} \mathcal{F}_{i}\left(\frac{t-t_{1}}{t_{K}-t_{1}}\right),
$$

see Fig. [7] This scaling law is of the class found in [9, 13].

Long times

The long times decay of the hump, i.e. for times well beyond $t_{K}$, is exponential

$$
\Delta \mathcal{E}(t)=a e^{-b t} .
$$

The curves $\Delta \mathcal{E}(t)$ for different $T_{1}$ can be made to collapse by shifting time according to

$$
t \rightarrow t+\left(t_{K}-t_{1}\right),
$$

that is to say that, the curves for the systems on which a temperature shift was applied are in advance with respect to the reference one.

Finally, we implemented the same protocol using temperatures $T_{1}$ and $T_{2}$ that are both below the dynamic critical temperature $T_{d}$. As shown in Fig. 9 the qualitative behaviour is the same as on the other side of the transition point. It is interesting to note that the curves for the perturbed system join, and become independent of $T_{1}$ before reaching the reference curve (this is similar to what observed in [8]).

In the low temperature phase the scalings discussed above are modified as follows. In the short-times regime, the scaling function (29) remains valid but the scaling form is modified to

$$
\begin{aligned}
\Delta \mathcal{E}(t) & =\left(T_{2}-T_{1}\right) \mathcal{F}_{s}\left(\left(t-t_{1}\right)\left(\frac{T_{1}}{T_{2}}\right)^{\alpha}\right), \\
\alpha & =\frac{T_{2}-T_{1}}{T_{d}} .
\end{aligned}
$$




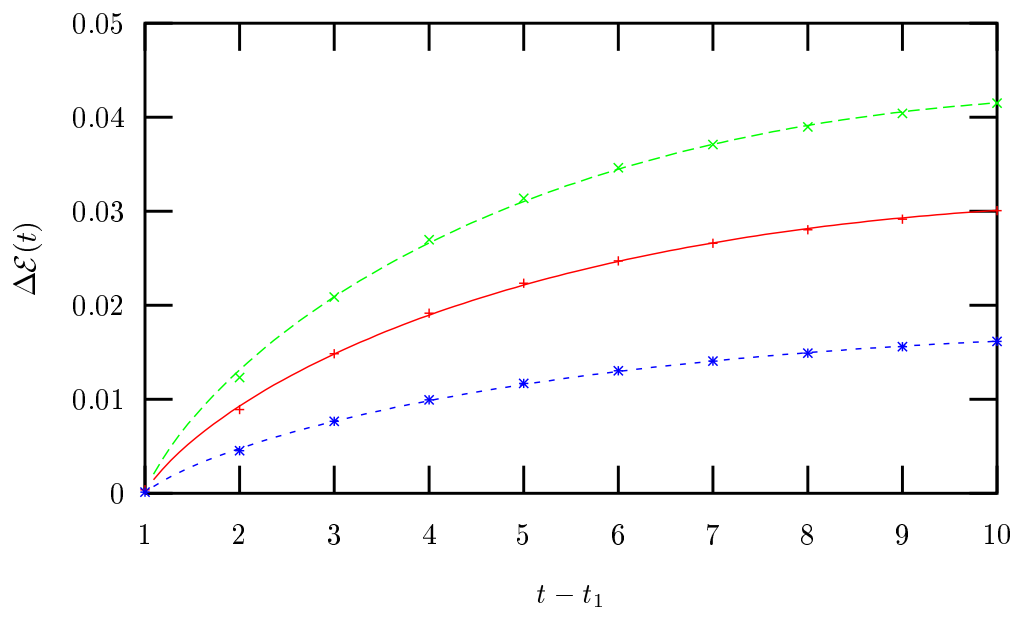

Figure 5: The short time fitting of the data for three values of the lower temperature, $T_{1}=0.6,0.65,0.7$, using Eq. 29).

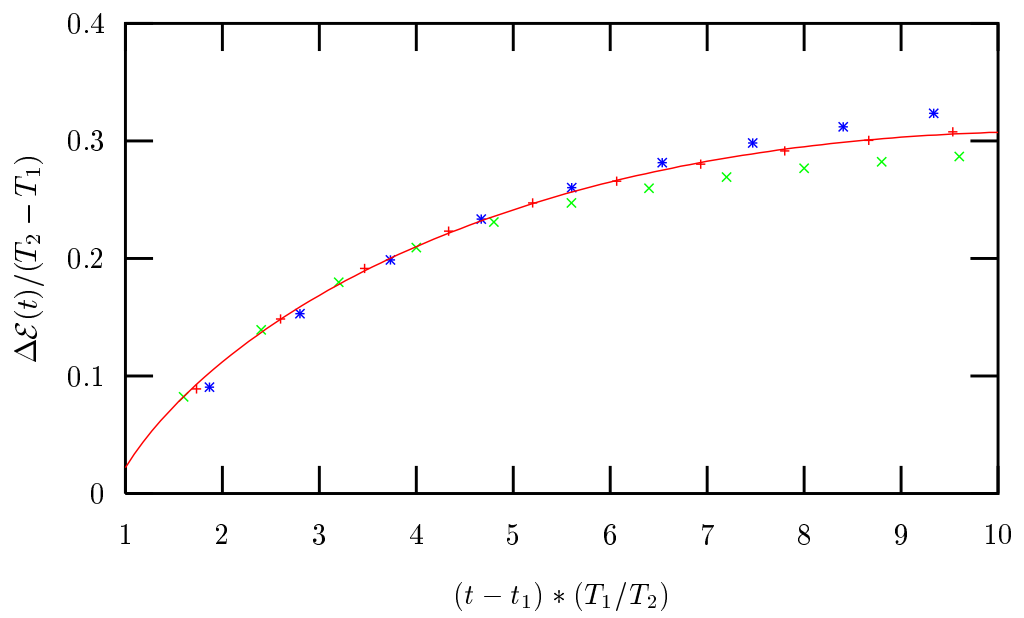

Figure 6: The short time scaling of the data for three values of the lower temperature, $T_{1}=0.6,0.65,0.7$, using Eq. (30). The solid line is a fit of the rescaled data for $T_{1}=0.65$ using Eq. (29) with $a=0.0623$ and $b=0.251$. 


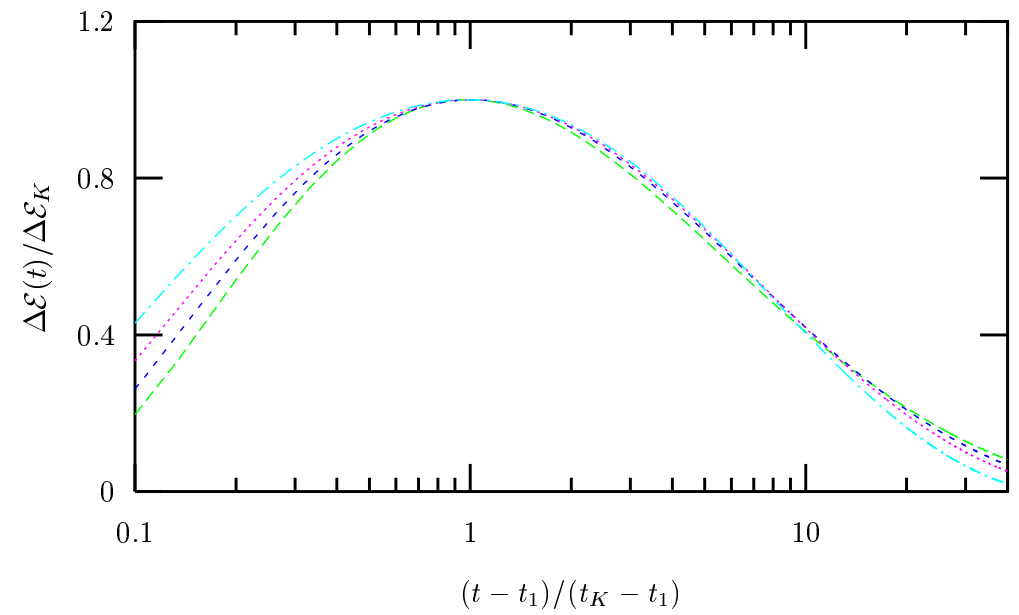

Figure 7: The scaling for intermediate times with $T_{1}=0.5,0.6,0.65,0.7$, see the text for an explanation.

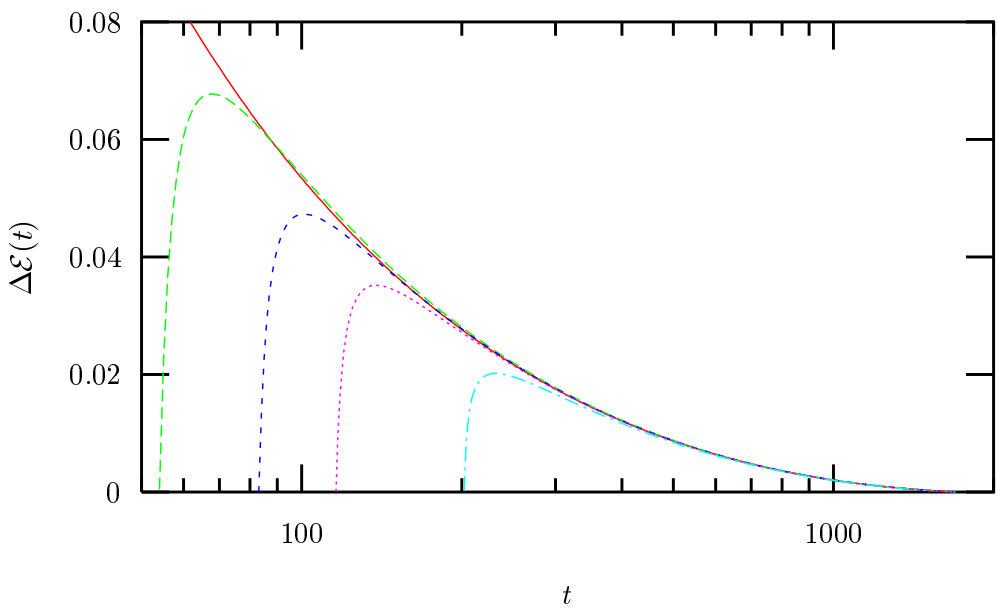

Figure 8: For long times the curves collapse on a master curve under the time-shift in Eq. (33). With red solid line the reference curve $\mathcal{E}^{\left(T_{2}\right)}(t)$; with dashed lines the curves under the effect of the temperature jump from $T_{1}=0.5,0.6,0.65,0.7$ translated in time $t \rightarrow t+\Delta t$ with $\Delta t=15,19,23,31$. The final approach to zero is exponential. 


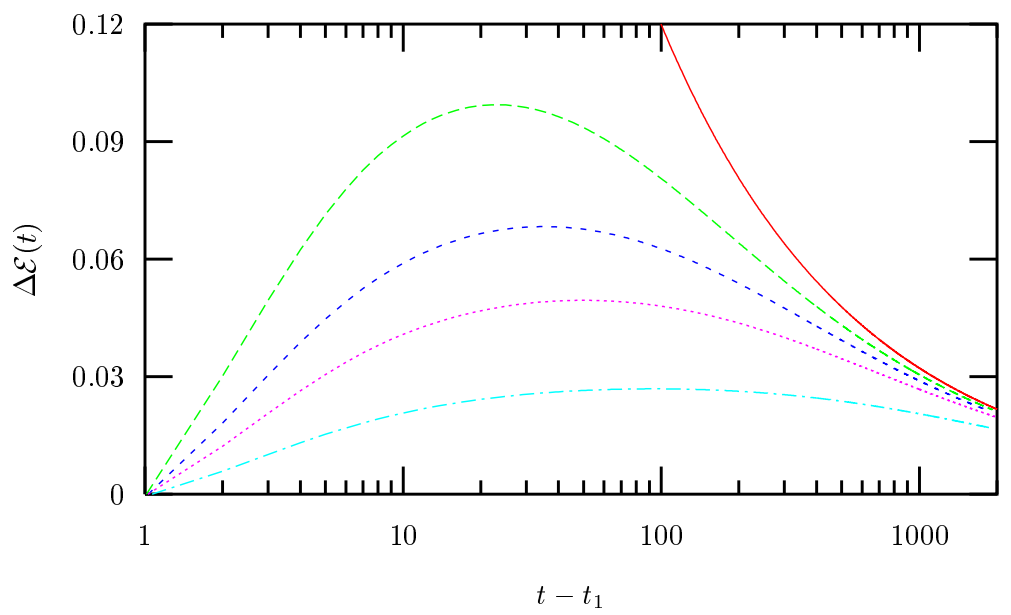

Figure 9: The hump in the glassy phase of the classical $p$-spin model with $p=3$ using $T_{1}=0.3,0.4,0.45,0.5$ with $t_{1}=80,173,330,1080$, respectively, and $T_{2}=0.55$.

For intermediate times we found

$$
\Delta \mathcal{E}(t)=\Delta \mathcal{E}_{K} \mathcal{F}_{i}\left(\left(\frac{t-t_{1}}{t_{K}-t_{1}}\right)^{1+\alpha}\right),
$$

with $\mathcal{F}_{i}$ independent of $T_{1}$ and $T_{2}$. At very long times, the approach to zero is a power law, $a t^{-b}$. The shift $t \rightarrow t+\left(t_{K}-t_{1}\right)$ is also efficient collapsing the data.

\subsection{Discussion}

The non-trivial content of the hump is its slow relaxation and dependence on $T_{2}-T_{1}$. The observed behaviour has been captured by several models already presented in the literature. Let us discuss these explanations and compare them to the one associated to the $p$ spin model.

First, one can compaire the behaviour observed in the super-cooled liquid phase with a simpler system: an overdamped harmonic oscillator. For this model a direct calculation yields

$$
\Delta \mathcal{E}(t)=\frac{1}{2}\left[k\left\langle x_{0}^{2}\right\rangle-k_{B} T_{1}\left(1-e^{\frac{2 k}{\gamma} t_{1}}\right)-k_{B} T_{2} e^{\frac{2 k}{\gamma} t_{1}}\right] e^{-2 \frac{k}{\gamma} t}
$$

with $k$ the harmonic constant, $\gamma$ the friction coeficient and $x_{0}$ the initial condition. The time $t_{1}$ is fixed by requiring that $\Delta \mathcal{E}\left(t_{1}\right)=0$. This condition forces the bracket in equation (37) to be zero and then, there is not a forthcoming hump. Clearly one 
needs to go beyond this model to get the observed two-temperature dependence and slow relaxation.

A simple next step is to study a model with a distribution of relaxation times that depends on temperature. A simple realization is the $2 d \mathrm{XY}$ model in the spin-wave approximation taking into account the contribution of vortices [11]. This model is given by a Gaussian free field (the angle of the local magnetization) with a $T$ dependent stiffness, $\rho(T)$. It has a slow dynamics characterised by the growth of a $T$ dependent correlation length $\ell_{T}(t)=(\rho(T) t)^{1 / 2}$ and it captures the phenomenology of the Kovacs' effect, as discussed by Berthier and Holdsworth [11].

In slightly more general terms [9, 12] the Kovacs' effect can be rationalized in any system with a growing temperature-dependent dynamic correlation length, that is shorter than the equilibrium one. When one shifts the temperature to $T_{2}$ at $t_{1}$, the length scales that are shorter than $\ell_{T_{1}}\left(t_{1}\right)$, and are hence equilibrated at $T_{1}$, have to reequilibrate at $T_{2}$, where their equilibrium energy is higher. The structure reached at $T_{1}$ has to break up and allow for the nucleation of new structures equilibrated at $T_{2}$. Instead, the length scales that are longer than $\ell_{T_{1}}\left(t_{1}\right)$ are still not equilibrated at $T_{1}$ and they may continue their evolution to equilibrate now at $T_{2}$. The former processes involve shorter length-scales and should be faster and dominate the first part of the relaxation after $t_{1}$ hence leading to an energy increase. The latter processes are slower and dominate the decay from the maximum towards the asymptotic value $\mathcal{E}_{a s}\left(T_{2}\right)$. Within this picture, the time at which the maximum in $\Delta \mathcal{E}$ is reached corresponds to the time when the small length scales have equilibrated at the new temperature $T_{2}$. A similar argument was put forward to explain the overshoot observed in the time-dependent dielectric constant of dipolar glasses after a temperature jump [3. It is also behind the calculation presented by Brawer on the ferromagnetic Ising chain at very low temperature [10].

However, it is not necessary to invoke a growing correlation length to capture the qualitative features of the Kovacs' effect. The main ingredient in the $p$-spin model that leads to this effect is the slow - non-exponential - and temperature dependent relaxation of the linear response after a strong perturbation. A scenario with a wide spectrum of relaxation times that depends on temperature was used in the past to explain the Kovacs' effect [5]. Here we demonstrated that, as one could have expected [12], the $p$-spin model or, equivalently, the MCT with no equilibration assumption, has this property.

The temperature and time dependence of the hump do depend on the model considered but the main qualitative features of the effect are shared by all of them. 
In the context of the spin models related to the MCT these will be obviously modified if one considers a mixture of $p=2$ and $p=4$ models (that corresponds to going from the schematic MCT to more refined versions).

Finally, let us discuss the relevance of the Kovacs' effect for the development of a thermodynamics of the nonequilibrium glassy state.

The attempts to use a thermodynamics for nonequilibrium glassy systems are based on the introduction of effective state variables, basically temperature and pressure. Initially, a (constant) fictive temperature that characterizes the glassy structure was introduced by Tool [30]. As a consequence of Kovacs' experiments it was realized that this single parameter was not enough to describe the evolution of the glass and the fictive temperature was upgraded to be a full history dependent function measuring the departure from equilibrium [1, 2, 31. Whether the fictive temperature, as defined in [1, 30, 31] behaves as a thermodynamic temperature remains to be proven. One can also translate the study of the Kovacs' effect in the parking lot model by Tarjus and Viot [14] in these terms: the second timedependent state variable introduced generalising Edwards' prescrition 32 acts as a fictive temperature.

More recently, an effective temperature was defined using the modification of the fluctuation - dissipation theorem in slowly evolving nonequilibrium systems. The interpretation of this quantity as a bonafide temperature was discussed, and some conditions on the physical relevance of this definition were also given [27]. In particular, the need to have a system evolving slowly - characterized by a slow relaxation of one time quantities - to be able to associate the properties of a temperature to the FDT ratio was reckoned and stressed. The situation in Kovacs' experiment goes beyond this limit: the system is strongly perturbed and the one-time quantity under study has a non-trivial, non-monotonic time-dependence. The Fluctuation Dissipation Relation also depends on time in a non-trivial manner [34]. Thus one cannot assert that it leads to a bonafide temperature and use it to construct a simple extension of thermodynamics to describe the subsequent behaviour of the system.

A similar conclusion, though expressed in terms of the potential energy landscape (PEL) scenario was reached by Mossa and Sciortino [8]. These authors showed, with molecular dynamic simulations of the fragile glass former OTP, that two systems in identical thermodynamic conditions (same values of $T, V, P$ ) can be in very different regions of their potential energy landscape if one of them has been strongly perturbed. Since the strongly perturbed system wanders in a region of the PEL that is never sampled in equilibrium, its configuration cannot be associated to that of 
an equilibrated liquid at a different temperature. The region of the PEL sampled allows for a definition of a microcanonical temperature only when the variation of the external conditions (temperature in this case) is small. Mossa and Sciortino arrived at this conclusion by comparing the properties of the inherent structures (closest local minimum in the PEL) visited during aging after a temperature jump of large magnitude to those sampled in equilibrium.

\section{Quantum model}

When quantum fluctuations are switched on one has to deal with the full SchwingerDyson equations (11)-(19). As explained in [33] the parameter that plays the rôle of the transverse field in trully quantum spin models is here the inverse of the mass $M$. Indeed, this model undergoes a phase transition in the $(T, \Gamma)$ plane with $\Gamma \equiv \hbar^{2} /(M \tilde{J})$ from an equilibrium paramagnetic phase to a non-equilibrium glassy one. In order to test the memory effect in the quantum problem, we then apply the Kovacs' protocol using $M^{-1}$ as the control parameter and we follow the evolution of the averaged potential energy density $\mathcal{E}(t)$.

In the quantum problem the asymptotic value of the potential energy density depends on $M$. (In the classical limit it does not.) The relaxation of the potential energy density at constant $M$ has (damped) oscillations whose magnitude depend on the parameter $M$. For large values of the mass the oscillations have a sufficiently large amplitude such that the asymptotic value falls within the oscillation, i.e. $\mathcal{E}(t)<\mathcal{E}_{a s}\left(T_{2}, \Gamma_{2}\right)$ for some finite times $t$. In the following we choose a value of $M_{2}$ such that the system is close to the paramagnetic - glass transition and for which $\mathcal{E}_{a s}\left(T_{2}, \Gamma_{2}\right)<\mathcal{E}(t)$ for all finite time $t$, see Fig. 10. Another feature to signal is that the oscillation in the energy-density decay may be such that there is more

than one value of $t_{1}$ for which $\mathcal{E}^{M_{1}}\left(t_{1}^{+}\right)=\mathcal{E}_{a s}^{M_{2}}$, see the curve drawn with a dashed line in Fig. 10 for which $M=0.8$.

Figure 11] shows the result of applying Kovacs' protocol to a system at $T=0.75$ with the reference value of the mass, $M_{2}=0.5$. We use four values of $M_{1}, M_{1}=0.6$, 0.8, 1 and 1.2 that satisfy $M_{1}>M_{2}$, i.e. $\Gamma_{1}<\Gamma_{2}$. For $M_{1}=0.6,1$ and 1.2 we find a unique $t_{1}$ satisfying $\mathcal{E}^{M_{1}}\left(t_{1}\right)=\mathcal{E}_{a s}^{M_{2}}$. For $M_{1}=0.8$ instead three solutions to this equation exist as shown in Fig. 10, Displayed in Fig. 11 are the reference energy density $\mathcal{E}^{\left(T_{2}, \Gamma_{2}\right)}(t)$ (solid red line) and the hump in the energy densities obtained by shifting the mass.

The first thing to note in the figure is that the curves depend on the value of $M_{1}$, 


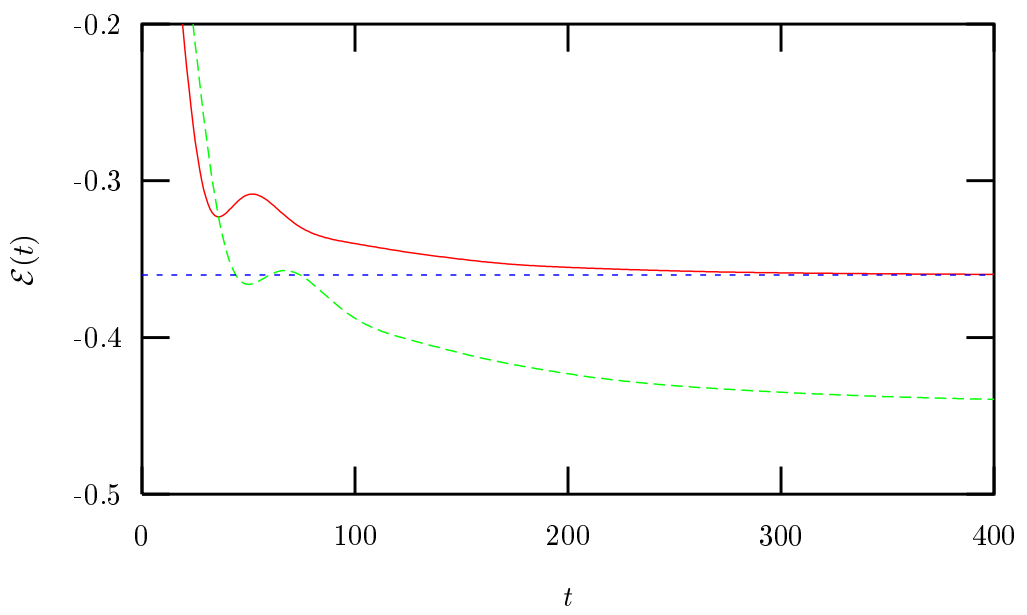

Figure 10: Energy-density decay in the quantum model with $p=3$ at constant mass and temperature. Solid line $M=0.5$ and dashed line $M=0.8$. In both cases $T=0.75$.

similarly to what happened in the classical case with $T_{1}$ : the larger the difference in the masses (or in the quantum parameter $\Gamma$ ), the more pronounced the effect. There is also a weak decreasing dependence of the position of the maximum with $M_{2}-M_{1}\left(\right.$ and $\left.\Gamma_{1}-\Gamma_{2}\right)$.

A second feature to remark is that the curves following the jump in $M$ go above the reference curve ( $c f r$. the classical problem where the approach to the reference curve always occurs from below) and also might have a negative initial part.

In the case in which there is more than one $t_{1}$, the form of the hump depends on the value chosen. In particular, there is no negative part in the hump if we take $t_{1}$ such that $\mathcal{E}^{\left(M_{1} \rightarrow M_{2}\right)}\left(t_{1}\right)$ is growing with $t$.

Finally, in the glassy phase $(T=0.2$ and $M=0.5)$ the oscillation in $\mathcal{E}(t)$ is almost completely damped. The hump has a very similar behaviour to the one found after a shift in $T$. Figure 12 shows the hump for several values of the pairs $\left(M_{1}, t_{1}\right)$ given in the caption. Also in this glassy case, the height of the hump increases with the difference $M_{2}-M_{1}$ and, simultaneously, the position of the maximum has a very smooth drift towards smaller values of $t-t_{1}$.

Thus, as far as the Kovacs' effect is concerned, we see that the quantum problem also shows a non-trivial dependence on the parameter $M_{1}\left(\Gamma_{1}\right)$ and a slow relaxation after the perturbation. 


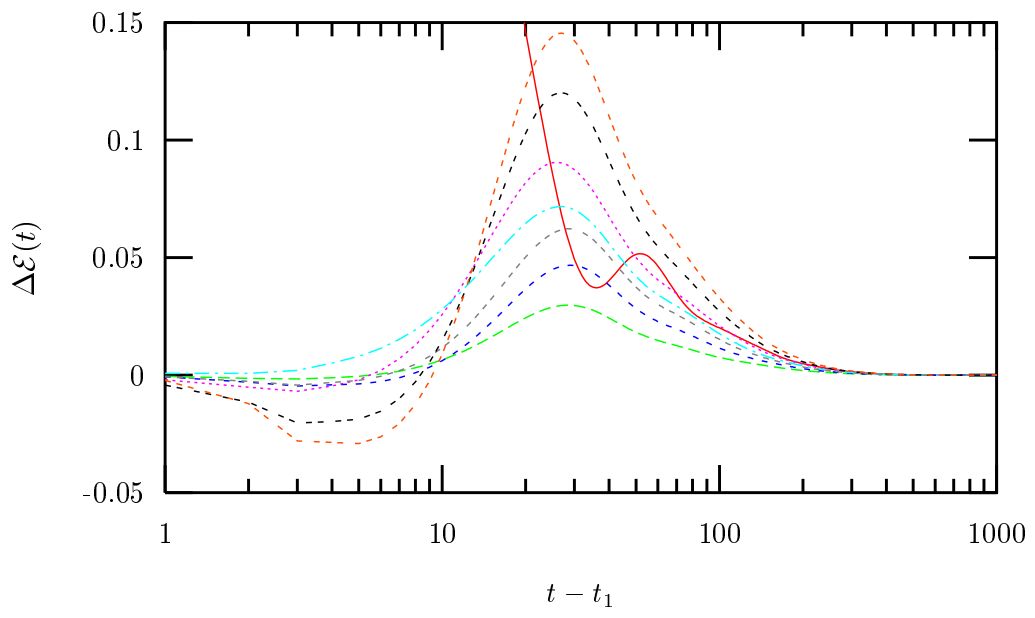

Figure 11: Hump in the quantum $p=3$ model at $T=0.75$. The reference curve, solid red line, corresponds to $M_{2}=0.5$. The modified curves have been obtained using, from bottom to top at the maximum, $M_{1}=0.6, t_{1}=95$ (dashed green); $M_{1}=0.7, t_{1}=82$ (dashed blue); $M_{1}=0.8, t_{1}=76$ (dashed gray); $M_{1}=0.8, t_{1}=62$ (dashed cyan); $M_{1}=0.8, t_{1}=46$ (dashed magenta); $M_{1}=1, t_{1}=47$ (dashed black) and $M_{1}=1.2, t_{1}=49$ (dashed orange).

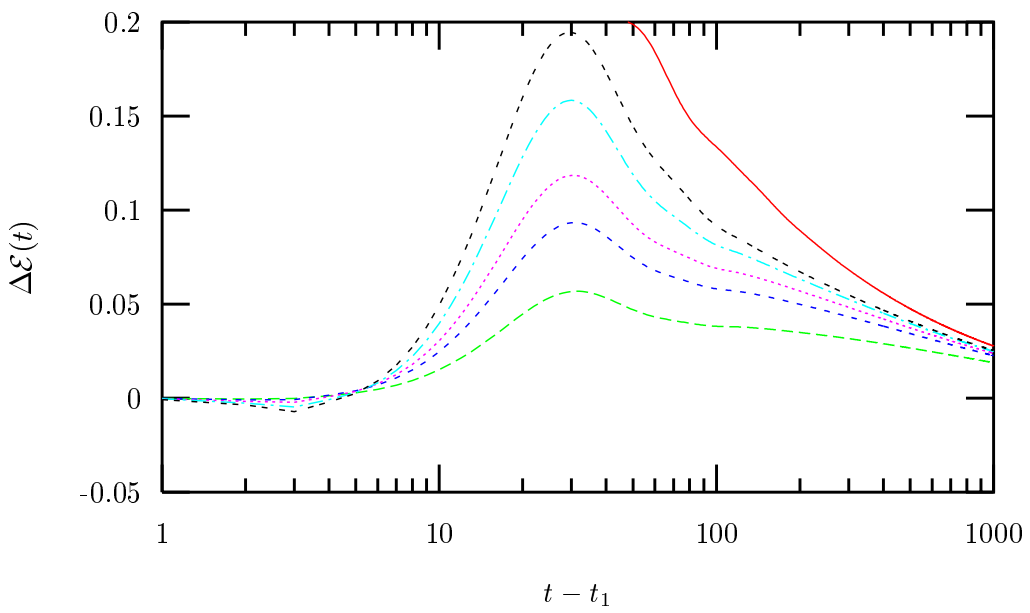

Figure 12: The hump as a function of the time-difference $t-t_{1}$ in the glassy phase of the quantum $p=3$ model using, from bottom to top, $M_{1}=0.6, t_{1}=539$ (dashed green), $M_{1}=0.7, t_{1}=274$ (dashed blue), $M_{1}=0.8, t_{1}=204$ (dashed magenta), $M_{1}=1, t_{1}=163$ (dashed cyan), $M_{1}=1.2, t_{1}=150$ (dashed black), and the reference curve at constant $M_{2}=0.5$ (solid red). The temperature is $T=0.2$ in all cases. 


\section{Conclusions}

We conclude that models with no spatial structure, like the $p$ spin spherical model that is intimately related to the schematic mode-coupling theory, can reproduce nontrivial memory effects when their non-equilibrium dynamics is studied. Similarly to what observed when reproducing the hole-burning protocol [18, we found here that the Kovacs' memory effect is captured by this model. In this sense, the Kovacs' experiment is not able to prove the existence of a growing correlation length in glassy systems. Assuming that a length scale exists one could, however, compare the outcome of this and other experiments to what can be derived from a domaingrowth like picture for glassy dynamics.

The reason for having these non-trivial long-memory effects in these fully - connected spin models is that close to their dynamic critical temperature (and below it) their response function after strong perturbation has been applied is not given by a simple exponential relaxation. The slow decay of the reponse implies that the effect of non-linear perturbations takes very long to disappear. This is encoded in the Schwinger-Dyson equations which describe the dynamical evolution of the system.

Acknowledegments We acknowledge financial support from the an Ecos-Sud travel grant and the ACI project "Optimisation algorithms and quantum disordered systems". LFC is research associate at ICTP Trieste and a fellow of the J. S. Guggenheim Foundation. G. S. L. is supported by CONICET. This research was supported in part by SECYT PICS 03-11609, PICS 03-05179 and by the National Science Foundation under Grant No. PHYS99-07949. We thank J-P Bouchaud and D. R. Grempel for very useful discussions.

\section{References}

[1] A. J. Kovacs, Adv. Polym. Sci (Fortschr. Hochpolym. Forsch.) 3, 394 (1963); A. J. Kovacs, J. J. Aklonis, J. M. Hutchinson and A. R. Ramos, J. of Pol. Sci. 17, 1097 (1979).

[2] G. Mc Kenna, J. Res. NIST 99, 169 (1984); Glass formation and glassy behavior in "Comprehensive polymer science" Vol. 12 (Polymer properties), C. Booth 
and C. Price eds. (Pergamon, Oxford, 1989) and refs. therein.

[3] F. Alberici-Kious, J-P Bouchaud, L. F. Cugliandolo, P. Doussineau, and A. Levelut, Phys. Rev. Lett. 81, 4987 (1998); Phys. Rev. B 62, 14766 (2000) .

[4] F. Lefloch, J. Hammann, M. Ocio, and E. Vincent, Europhys. Lett. 18, 647 (1992). M. Sasaki, V. Dupuis, J-P Bouchaud, and E. Vincent, Eur. Phys. J. B 29, 469 (2002).

[5] L. C. E. Struik, Aging in amorphous polymers and other materials (Elsevier, Amsterdam) 1978.

[6] R. L. Leheny and S. R. Nagel, Phys. Rev. B 57, 5154 (1998). R. Höhler, S. Cohen-Addad and A. Asnacios, Europhys. Lett. 48, 93 (1999). L. Bellon, S. Ciliberto, and C. Laroche, Europhys. Lett. 51, 551 (2001). O. Kircher and R. Böhmer, Eur. Phys. J. B 26, 329 (2002). F. Ozon et al, cond-mat/0210554. A. Parker and V. Normand, cond-mat/0306056.

[7] C. Josserand, A. V. Thachenko, D. M. Mueth, and H. M. Jaeger, Phys. Rev. Lett. 85, 3632 (2000).

[8] S. Mossa and F. Sciortino, Phys. rev. Lett. 92, 045504-1 (2004)

[9] L. Berthier and J-P Bouchaud, Phys. Rev. B 66, 054404 (2002).

[10] S. Brawer, Phys. and Chem. of Glasses 19, 48 (1978).

[11] L. Berthier and P. C. W. Holdsworth, Europhys. Lett. 58, 35 (2002).

[12] E. Bertin, J.-P. Bouchaud, J.-M. Drouffe, C. Godreche, J. Phys. A: Math. Gen. 36, 10701 (2003).

[13] A. Buhot, J. Phys. A 36, 12367 (2003).

[14] G. Tarjus and P. Viot, Memory and Kovacs effects in the parking-lot model: an approximate statistical-mechanical treatment, cond-mat/0310738, Proceedings from "Unifying concepts in granular media and glasses", Capri 2003.

[15] A. Crisanti and H-J Sommers, Z. Phys. B 87, 341 (1992).

[16] L. F. Cugliandolo and J. Kurchan, Phys. Rev. Lett. 71, 173 (1993). 
[17] L. F. Cugliandolo, cond-mat/0210312, Lecture notes in "Slow Relaxation and non equilibrium dynamics in condensed matter", Les Houches Session 77, J-L Barrat et al Eds., (EDP-Springer, 2003).

[18] L. F. Cugliandolo and J. L. Iguain, Phys. Rev. Lett. 85, 3448 (2000), ibid 87, 129603 (2001). G. Diezemann, Phys. Rev. E 68 021105, 2003. U. Hberle, G. Diezemann, Nonresonant holeburning in the Terahertz range: Brownian oscillator model cond-mat/0308056.

[19] See, e.g. R. Richert, J. Phys. C 14 R703 (2002) and references therein.

[20] W. Wu, B. Ellmann, T. F. Rosenbaum, G. Aeppli, and D. H. Reich, Phys. Rev. Lett. 672076 (1991); W. Wu, D. Bitko, T. F. Rosenbaum, and G. Aeppli, Phys. Rev. Lett. 711919 (1993).

[21] S. Ludwig and D. D. Osheroff, Phys. Rev. Lett. 91, 105501 (2003). S. Ludwig, P. Nalbach, D. Rosenberg, and D. D. Osheroff, Phys. Rev. Lett. 90, 105501 (2003). S. Rogge, D. Natelson, and D. D. Osheroff, Phys. Rev. Lett. 76, 3136 (1996).

[22] Z. Ovadyahu and M. Pollak, Phys. Rev. B 68, 184204 (2003). V. Orlyanchik and Z. Ovadyahu, Phys. Rev. Lett. 92, 066801 (2004). A. Vaknin, Z. Ovadyahu and M. Pollak, Phys. Rev. B 65, 134208 (2002).

[23] L. F. Cugliandolo and G. Lozano, Phys. Rev. Lett. 80, 4979 (1998), Phys. Rev. B 59, 915 (1999). L. F. Cugliandolo, D. R. Grempel, G. Lozano, H. Lozza and C. A. da Silva Santos, Phys. Rev. B 66, 014444 (2002).

[24] L. F. Cugliandolo, D. R. Grempel, G. Lozano and H. Lozza, cond-mat/0312064.

[25] M. Rokni and P. Chandra, Phys. Rev. B 69, 094403 (2004). R. Serral Gracias and T. M. Nieuwenhuizen, cond-mat/0304150.

[26] W. Götze, in Liquids, freezing and glass transition, Les Houches 1989, JP Hansen, D. Levesque, J Zinn-Justin eds. North Holland. W. Götze and L. Sjögren, Rep. Prog. Phys. 55, 241 (1992).

[27] L. F. Cugliandolo, J. Kurchan and L. Peliti, Phys. Rev. E 55, 3898 (1997).

[28] L. F. Cugliandolo and D. S. Dean, J. Phys. A 28, 4213 (1995). 
[29] L. F. Cugliandolo and J. Kurchan, Phys. Rev. B 60, 922 (1999).

[30] A. Q. Tool, J. Am. Ceram. Soc. 29, 240 (1946).

[31] O. S. Narayanaswamy, J. Am. Ceram. Soc. 54, 491 (1971).

[32] S. F. Edwards, in Granular Matter; an interdisciplinary approach, A. Mehta ed. (Springer-Verlag, New York, 1994). A. Mehta and S. F. Edwards, Physica A 157, 1091 (1989). S. F. Edwards amd R. Oakeshott, Physica A 157, 1080 (1989).

[33] L. F. Cugliandolo, D. R. Grempel and C. A. da Silva Santos, Phys. Rev. Lett. 85, 2589 (2000), Phys. Rev. B 64, 014403 (2001).

[34] Note the surprising result in [13] for two kinetically constrained models. 